WHITE PAPER TO THE NRC DECADAL SURVEY

Giant Planet Systems and Technology Development Topic Areas

\title{
TPS and Entry Technologies for Future Outer Planet Exploration
}

\author{
By \\ Donald Ellerby (Donald.T.Ellerby@nasa.gov) (650) 380-8957) \\ Helen Hwang, Matt Gasch, Robin Beck, Todd White \\ NASA Ames Research Center, Moffett Field, CA 94035
}

\begin{abstract}
Co-Signers (51 individuals from 18 organizations): Aaron Brandis, David Driver, Kristina Skokova (Analytical Mechanics Associates, Inc.); Douglas Adams, Kenneth Hibbard (Johns Hopkins Applied Physics Laboratory); Amanda Brecht, David Hash, Cole Kazemba, Dean Kontinos, Milad Mahzari, Mary Kathleen McGuire, Suman Muppidi, Harry Partridge, Margaret Stackpoole, Ethiraj Venkatapathy, Michael J. Wright, Kerry Zarchi (NASA Ames Research Center); Michele Chaffey, Mary Litwinski (Boeing Research and Technology); Mark Lippold, Steven Violette (Fiber Materials, Inc.); Conor Nixon, Melissa Trainer (NASA Goddard Space Flight Center); Thomas Spilker (independent consultant); David Atkinson, Alexander Austin, Kevin Baines, Tibor Balint, Bernard Bienstock, James Cutts, Jeffery L. Hall, Siddharth Krishnamoorthy, Kim Reh, Eric Slimko, Linda Spilker (NASA Jet Propulsion Laboratory); Adam Amar, Stan Bouslog, Gerald Lebeau, Steven Rickman (NASA Johnson Space Center); Ye Lu (Kent State University); Alicia Dwyer-Cianciolo, Soumyo Dutta, N. Ronald Merski, Michelle Munk (NASA Langley Research Center); Jarvis Songer (Lockheed Martin Space); Manish Mehta (NASA Marshall Space Flight Center); Paul Byrne (North Carolina State University); Matthew Kelly (Peraton); Kandis-Lea Jessup (Southwest Research Institute); Sushil Atreya (University of Michigan); Martha Gilmore (Wesleyan University)
\end{abstract}

\begin{abstract}
The lack of knowledge about the Ice Giants (Voyager being the only probe to briefly visit them) and literally narrow window of planetary alignment for pragmatic missions to the Ice Giants, make them high priority targets in the next decade. The purpose of this white paper is to provide an overview to the NRC Decadal Survey on thermal protection system (TPS) technologies required for future Outer Planet exploration missions. A prime conclusion is that several of the most significant recommendations made during the last decadal survey were addressed positioning the TPS community to better support missions to the Outer Planets. These include maturation of the Heatshield for Extreme Entry Environment Technology (HEEET) to TRL 6 as replacement for Carbon Phenolic (that requires requalification itself), TPS test facility upgrades, and design tool improvements. These new materials and test methods are enabling for Outer Planet missions. However, there are limitations in the HEEET technology and available ground based test facilities that could become mission constraints depending on the science objectives. This white paper discusses opportunities to mitigate those constraints. In addition, if a mission is not scheduled in the near future, these new developments are at risk of becoming unavailable. Therefore, we recommend that NASA invest in a cross-cutting technology program that focuses on sustainment of relevant TPS materials, entry systems, test facilities, design tools, and flight instrumentation.
\end{abstract}




\section{INTRODUCTION}

This NRC Decadal Survey white paper, provided by the thermal protection technology community, is a general assessment of the current capability of thermal protection systems (TPS) with respect to the scientific exploration of the Outer Planets (OP), as well as anticipated TPS requirements in support of future OP missions. The paper begins with a brief history of thermal protection systems relevant to the exploration of the OP and follows with a discussion of current TPS material, test, and analysis capabilities. Potential limitations in these areas, based on mission needs to meet science objectives, are described and recommendations are presented for establishing a TPS Technology Program that includes development, testing, and manufacturing capabilities to support future OP missions. The maturation of Heatshield for Extreme Entry Environments Technology (HEEET) and upgrades to ground based test facilities and analysis techniques have addressed the key limitations identified in the previous decadal survey Outer Planet and Venus white papers [1,2](Venus has relevant entry conditions to OP missions). Mission studies $[3,4]$ have shown that these new capabilities can support missions to each of the OP destinations. However, known technology and testing limitations are identified in this paper, along with recommendations to mitigate mission constraints. In the context of this document, the Outer Planets refers to Saturn, Uranus and Neptune. The entry conditions at Jupiter are in a different regime posing unique challenges compared to other destinations and therefore are not covered in this white paper. Currently available forebody TPS materials cannot meet the requirements of entry missions at Jupiter, which would require requalification of Carbon Phenolic (CP), or development of new TPS materials. This paper is focused on direct entry missions to the OP. However, aerocapture offers distinct advantages over direct entry missions. Aerocapture details are presented in the Aerocapture as an Option for Ice Giants Missions decadal white paper [5] and the Thermal Protection System to Enable Ice Giant Aerocapture Mission for Delivering Both an Orbiter and an In-Situ Probe [6], which provide a discussion of these opportunities.

\section{BACKGROUND}

The previous OP TPS decadal white paper [1] and the current Technologies for Future Venus Exploration decadal white paper [7] provide a good historical overview of the TPS systems that have previously been evaluated for use in OP missions. In short, the previous decadal white paper identified the absence of a flight-qualified TPS for OP entry conditions as a key limitation for the implementation of those missions and recommended NASA invest in the requalification of the full density heritage Carbon Phenolic (HCP), or invest in the development of a more mass efficient and sustainable alternative material. NASA selected the latter option and invested in developing and maturing HEEET to TRL 6. In addition, the previous decadal white paper identified groundbased testing limitations which have partially been addressed by NASA's investment in upgrades to the Ames Research Center's (ARC) Interaction Heating Facility (IHF) arcjet.

The current Venus white paper [7] and the Sustaining Mature Thermal Protection Systems Crucial for Future In-Situ Planetary Missions white paper [8] address the challenges with continued sustainment of entry systems technologies in the medium to long-term (10+ years), given the infrequent rate of missions requiring entry systems technologies, the unique-to-NASA requirements for many of the technologies, and the extreme Venus and OP conditions. Investments in sustaining these technologies are critical for maintaining the ability to implement missions to the OP in the next decade, and avoid the lack of qualified materials as experienced in the recent past. 


\section{CURRENT CAPABILITY: Thermal Protection Systems \\ Materials}

Given the properties of the OP atmospheres $\left(\mathrm{H}_{2} / \mathrm{He}\right)$ and the expected entry velocities for OP probes, the forebody thermal protection systems for OP entry vehicles will require use of ablative materials.

Table 1 lists the capabilities of currently available flight-proven TPS materials in the United States as well as potential performance limits for new TPS materials that have been developed since the previous white paper. There are materials not included in the table that are at lower TRLs, are developed outside of the United States, do not have widely available performance data, or have not been specifically evaluated for these entry applications.

Table 1. Candidate Forebody and Backshell TPS materials for Direct Entry Outer Planet probes.

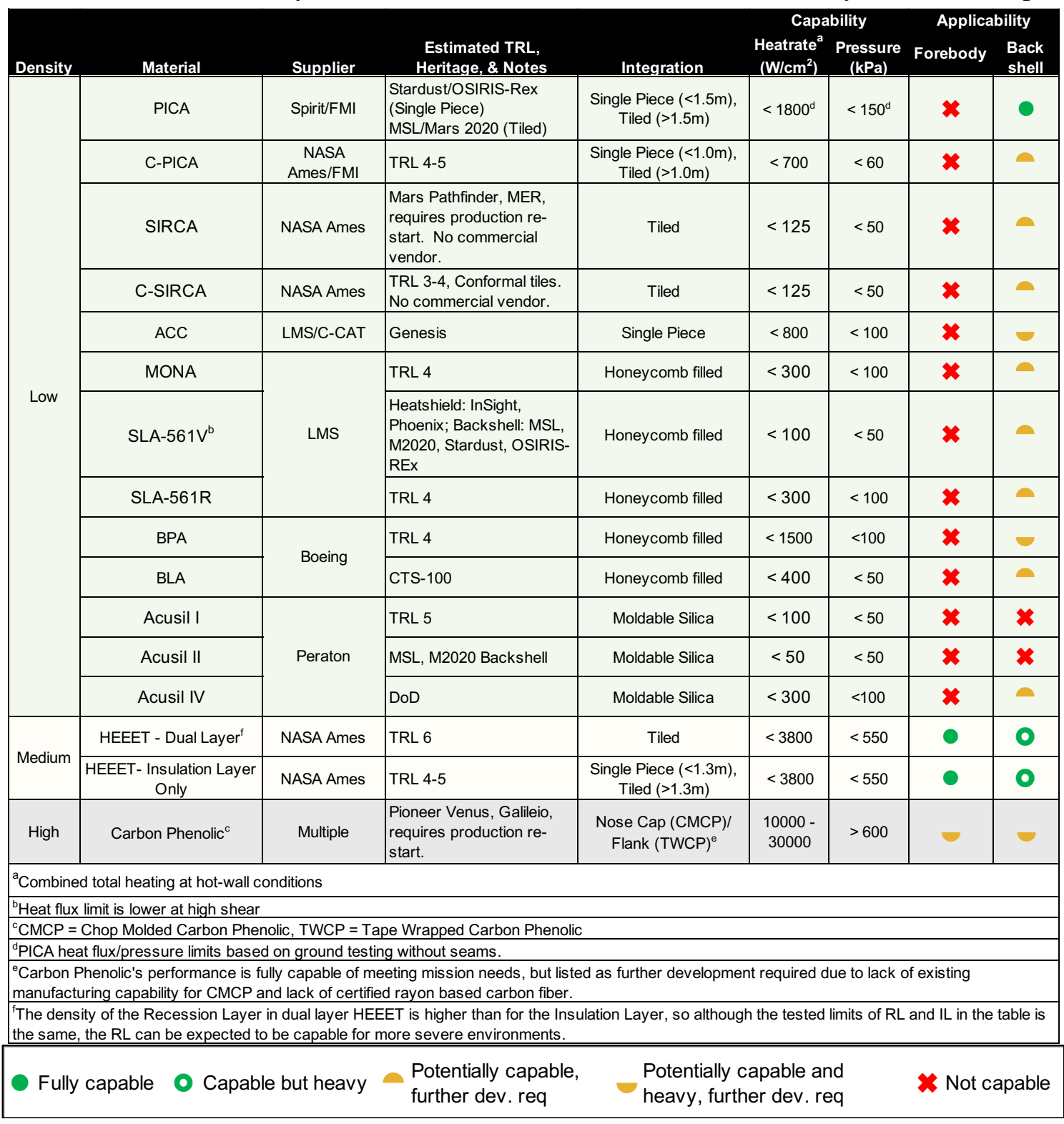


Table 1 illustrates applicability of forebody TPS candidates for direct entry OP probe missions. Given the extreme entry environments at the OP only the mid-density and high density materials are applicable for forebody heat shield applications.

Although Chop-Molded HCP was critical to the Pioneer Venus and Galileo missions, it has not been produced for flight since the 1980s. NASA Ames held two workshops (FY10 and FY11) concerning the supply chain issues with respect to HCP and proposed the development work required to qualify a new rayon-based carbon fiber as well as a proposed alternate, more sustainable, material to HCP. Ultimately NASA deemed the restart of HCP as not sustainable and chose to invest in the Heatshield for Extreme Entry Environment Technology (HEEET) material instead. The HEEET dual-layer design, shown in Fig. 1 (x-ray computerized tomography (CT) scan), is a 3-D woven material [3] and is more mass efficient than HCP for missions that use high speed entry to deliver landers, probes, aerial platforms, higher speed skimmers, and aerocapture.
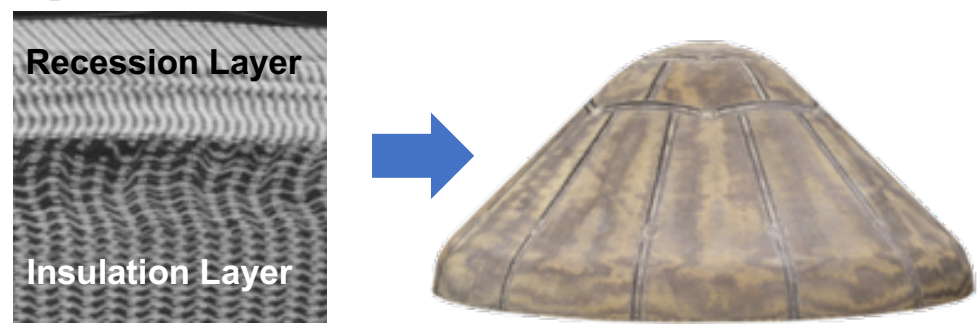

Figure 1. CT scan of HEEET dual layer (left) and 1-meter Engineering Test Unit (right).

Table 1 also lists material capabilities for usage over the entire backshell, even in regions of reattaching flow (moderate heat fluxes and shear forces). It is possible to use several materials, particularly low-density materials, in lower heating areas. There may also be regions on the backshell where radio frequency (RF) transparent TPS materials will be required to allow communications, and a number of candidate materials are available to meet this requirement as well (ex. Acusil II).

Table 2 presents ranges of potential stagnation point environments for direct entry missions to Saturn, Uranus, and Neptune for comparison purposes. There is a broad range of potential entry environments depending on mission design parameters such as: entry velocity, entry flight path angle, probe ballistic coefficient (size, shape, mass), target latitude, and prograde or retrograde entry. This table should not be interpreted as encompassing all potential environments. A key lessons from previous mission concepts was that the stagnation point (listed in this table) may not result in the highest heat fluxes on the heat shield. Roughness-augmented turbulent heating was often found to create the highest entry heating conditions on the flank of the vehicle. It is worth noting that the total heat load will determine the TPS thickness required, whereas the peak heat flux and peak pressure determines if the material may undergo failure modes. Bottom line is that for each destination there are options within the design space that are achievable with current capabilities, as seen in comparions of HEEET test points with entry environments in Fig. 2.

Table 2. Potential stagnation point environments for direct entry missions

\begin{tabular}{|c|c|c|}
\hline Destination & $\begin{array}{c}\text { Stagnation Point Heat } \\
\text { Flux }\left(\mathbf{W} / \mathbf{c m}^{\mathbf{2}}\right)\end{array}$ & $\begin{array}{c}\text { Stagnation Point } \\
\text { Pressure (kPa) }\end{array}$ \\
\hline Saturn & $2000-8000$ & $150-900$ \\
\hline Uranus & $1800-3000$ & $220-1000$ \\
\hline Neptune & $1500-2500$ & $200-1200$ \\
\hline
\end{tabular}




\section{Ground Test Facilities}

The main ground-test facilities for TPS development for the past several decades have been the high-enthalpy arcjet facilities at ARC, Arnold Engineering and Development Center (AEDC), and Boeing Large Core Arc Tunnel (LCAT). These facilities have power capabilities from 10 to $60 \mathrm{MW}$, provide the largest test article or the highest heating capability possible, and have been used for TPS development work, as well as qualification of flight materials. In parallel with the HEEET development, the test facilities at ARC were upgraded with the addition of a new 3-inch diameter nozzle, capable of achieving peak conditions of $\sim 4,000 \mathrm{~W} / \mathrm{cm}^{2}$ heat flux at $\sim 500 \mathrm{kPa}$ pressure on $25 \mathrm{~mm}$ diameter test articles. Figure 2 provides a comparison between test facility capabilities in relation to potential OP entry environments showing that for each destination there are feasible mission design parameters.

With the addition of the 3-inch nozzle in IHF, arcjet facilities are now capable of simulating the combined high heat fluxes and high pressures associated with hyperbolic direct entry to OP destinations, although there are mission scenarios that result in environments outside the ground testable range. The Laser Hardened Materials Evaluation Laboratory (LHMEL) facility at the Air Force Research Laboratory at Wright Patterson Air Force Base has supported the aerospace community for several decades. LHMEL has both a 10-kW and 100-kW carbon dioxide (10.6$\mu \mathrm{m})$, continuous wave, flat top laser. The LHMEL facility allows testing of TPS materials at the estimated heat flux required to initiate char spallation, but only at atmospheric pressure. The HEEET system was tested at LHMEL to $8000 \mathrm{~W} / \mathrm{cm}^{2}$ without any issues, whereas traditional Tape Wrapped and Chop Molded HCP samples cracked and demonstrated ply separation. It is important to note that none of these facilities listed here have the capability to test in the $\mathrm{H}_{2} / \mathrm{He}$ atmosphere of the outer planets.

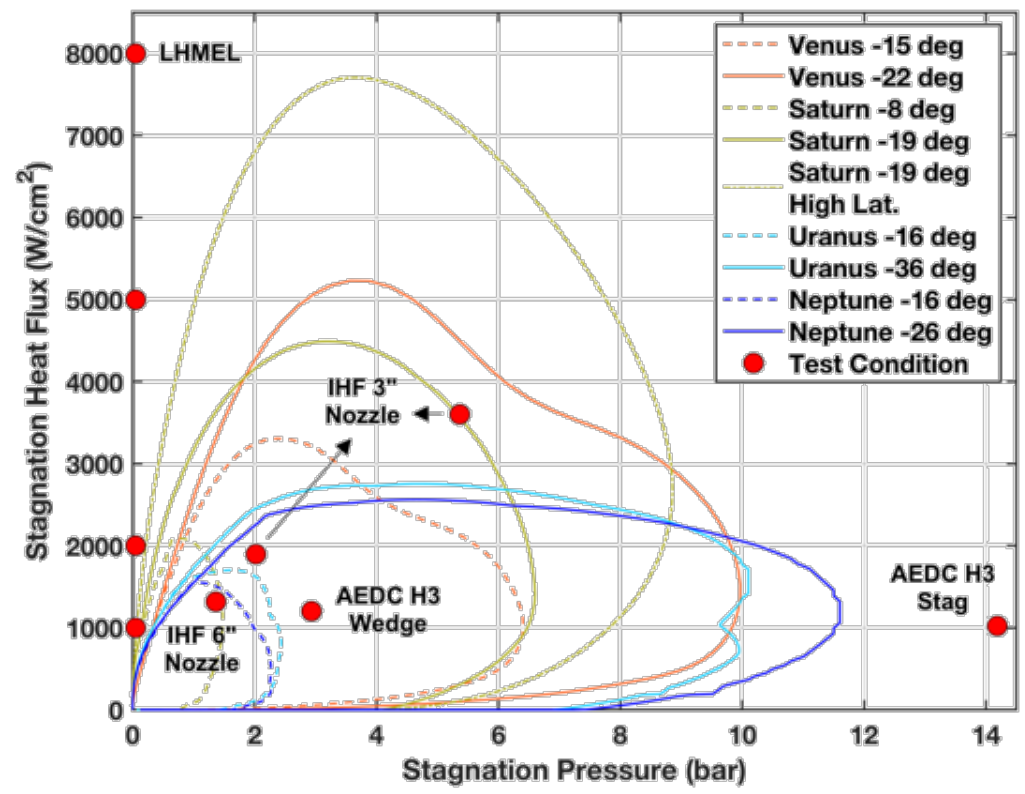

Figure 2. Arcjet envelopes for Outer Planet and Venus destinations at different entry flight path angles, overlaid with relevant test facility capabilities (red circles).

\section{ISSUES AND CHALLENGES FOR TPS AT OUTER PLANETS}

Materials

There are several TPS options that have been qualified for the expected backshell environments of OP missions, however these materials may not be mass efficient in the required $100-300 \mathrm{~W} / \mathrm{cm}^{2}$ heating range. Because the OP probes are generally designed to be small (base diameters $<3 \mathrm{~m}$ ), 
therefore a slight increase in backshell mass could lead to large inefficiences. Every extra kilogram of savings on the backshell could mean an extra kilogram of payload, so there is incentive to develop more mass efficient options that would be robust to potential flow reattachment which would drive down the heating range.

For the heatshield, the OP entry heating is expected to be higher than that for Venus and with higher total heat loads. Mission studies have shown that HEEET, in its current configuration, satisfies requirements at each destination. However, combinations of mission design parameters (as discussed previously) can lead to conditions that would result in HEEET thicknesses beyond the currently qualified limit of 2", including 0.6 " of Recession Layer (RL).

Choices of mission design parameters can also push entry environments beyond the range for which the materials are currently qualified. This is illustrated in comparing the heat fluxes and pressure ranges in Table 2 and Figure 1 to that for which material capability has been demonstrated in Table 1. For example, some Saturn mission concepts have stagnation heat fluxes of $8000 \mathrm{~W} / \mathrm{cm}^{2}$. However, by carefully selecting mission parameters the entry environments can be reduced such that existing TPS materials can be used for each of the OP destinations, with the exception of Jupiter. Material constraints are discussed in the following sections.

\section{HEEET: Maximum Woven Thickness}

There are multiple aspects that determine the maximum woven thickness for HEEET. One limiting factor of the woven thickness is the loom design. Another is that the HEEET seam design is dependent on the TPS thickness. The HEEET project demonstrated manufacturing and performance for dual layer thickness of $\sim 0.6$ " RL and $\sim 1.4$ " of Insulating Layer (IL). The details of the seam integration approach depend on the RL thickness and the ratio of RL to IL thickness. Further seam development is required if there is a significant deviation from the previously demonstrated RL thickness ( 0.6") or RL to IL ratio (0.6":1.4"). Past OP mission studies have indicated that RL thickness could be greater than 0.6 " and may have significantly different RL:IL ratios warranting further development. In order to avoid a large investment in developing thicker RLs or higher ratios of RL:IL, mission design choices can be made to reduce entry environments.

\section{HEEET: Eliminating Gap Fillers}

The seams are the area of largest risk for the material, both aerothermally and structurally. By eliminating the gap fillers, the system would become more robust and could potentially be used in environments beyond those tested in existing ground facilities. Currently, the HEEET loom is capable of weaving 24" width material. By increasing the width to 80 ", it may be possible to form a single piece heatshield (without gap fillers) of IL-only material. This width would support a 52 " diameter probe. The Mars Sample Return Earth Entry Vehicle project is currently investigating this approach, with a derivative of HEEET refered to as 3D Woven Mid-Density Carbon Phenolic (3MDCP), which would be able to weave HEEET IL up to 1.4 inches in thickness. However this may be too thin for some OP missions. The HEEET IL has been tested at the same extreme environments (except the $14 \mathrm{~atm}$ test point shown in Fig. 2) as the dual layer material. Both dual layer and single layer materials performed well with good comparisons of measured versus predicted recessions, indicating there are no failures in the IL-layer-only material in these environments. However, the higher density of the RL layer make it a more robust material, capable of higher entry conditions.

\section{Ground Test Facilities}

The last ten years have seen a significant improvement in the ground testing capabilities, particularly with the addition of the 3-inch nozzle in the IHF arcjet facility. HEEET dual layers and IL-only have been tested up to $\sim 3500 \mathrm{~W} / \mathrm{cm}^{2}$ and $\sim 5$ atm with predictable recession and no 
failures. In addition, HEEET has been tested for both the dual layer and IL-only in the AEDC H3 facility with an applied shear of $\sim 4000 \mathrm{~Pa}$, a heat flux of $\sim 1200 \mathrm{~W} / \mathrm{cm}^{2}$, and a pressure of $>200$ $\mathrm{kPa}$ with no failures and recession rates that match the predicted values from thermal response models that include turbulent roughness-augmented heating.

Due to facility limitations, all arcjet testing to date has been in air. It is likely that the oxidizing air environment in test is more aggressive than the relatively inert $\mathrm{H}_{2} / \mathrm{He} \mathrm{OP}$ atmospheric mixtures. Therefore, failure modes are more likely to occur in air. The margins applied to the calculated thicknesses required for HEEET are assumed to be sufficient to accommodate any discrepancies due to the $\mathrm{H}_{2} / \mathrm{He}$ atmosphere. These assumptions have not been verified by test, and existing test facilities cannot achieve OP-relevant environments in $\mathrm{H}_{2} / \mathrm{He}$. Testing in $\mathrm{N}_{2}$ at $\mathrm{OP}$ conditions is feasible and a reasonable substitute, given the lack of an existing $\mathrm{H}_{2} / \mathrm{He}$ facility.

Some of the OP mission concepts, with steep entry flight path angles, drive the pressures very high, beyond the testable range. High pressures are a risk because the material may fail due to material spallation (behaviour not modelled with current tools). Currently, mission designs are limited to pressures below $600 \mathrm{kPa}$ (shallower entries) which may impact the desired science objectives. Figure 2 shows a test point at $\sim 14$ atm at AEDC, however, during testing at this condition both HCP and HEEET showed substantially higher recession rates than predicted. The root cause of the discrepancy is not known. Videos of the testing do not show any obvious failure modes so it is unknown if the discrepancy is due to a test article effect, error in the estimates of the facility environment, a material response modeling error, or a failure that is not obvious in the video images.

A more thorough discussion on ground test facilities needs relevant for OP missions can be found in the Ground Test Facilities for Future Atmospheric Entry white paper [9].

\section{RECOMMENDATIONS}

It is recommended that NASA establish a cross-cutting TPS Technology program with elements focused on enabling OP missions. The program will need to focus on the following:

Materials:

1. Ensure the fabrication of TPS materials for extreme environments is maintained by conducting annual or semi-annual manufacturing assessments

2. Expand the capability of HEEET by increasing woven thickness and developing single piece construction heat shields (including loom upgrades) and gapfillers for much thicker heat shields

3. Sustain current manufacturing capabilities for existing and heritage TPS materials for at least two proven backshell materials to be available for future OP missions

\section{Test facilities:}

1. Ensure the ability to test in extreme environments at existing facilities (such as arcjets at ARC and AEDC) is maintained at very high heat fluxes $\left(\geq 4 \mathrm{~kW} / \mathrm{cm}^{2}\right)$

2. Endeavour to provide the capability for testing in $\mathrm{N}_{2}$ which represents a relatively small investment, and potentially $\mathrm{H}_{2} / \mathrm{He}$, which is much more expensive.

3. Evaluating the effects of surface roughness on turbulent heating $[10,11]$

\section{Analytic and Model Development:}

Continue to improve design and analysis tools, such as CFD and material response models, needed to verify material response and qualification test conditions. Thermochemistry effects and heating augmentation due to surface roughness also need to be modeled. Improve understanding of TPS failure modes through modeling in hope of extrapolating to higher environments, predicting response differences in $\mathrm{H}_{2} / \mathrm{He}$, and/or explaining observed testing artifacts 


\section{Flight Instrumentation:}

Any future OP mission will need embedded instrumentation to verify the performance of HEEET, which has not been flown. Similar to the Mars entry instrumentation sensors that were a part of Mars Science Laboratory and Mars 2020 [12], thermocouple plugs need to be developed for HEEET. These plugs measure near-surface and in-depth thermal performance of the TPS, which will help to verify material and gapfiller performance during flight. This will be critical for understanding and improving the TPS for subsequent missions.

In conclusion, it is worth reinforcing that studies to date have shown that, although constrained by current capabilities, there are mission designs for each of the Outer Planets (except Jupiter) that are feasible with existing TPS materials and ground test facilities. The recommendations outlined in this paper, if implemented, have direct benefit to other planetary missions, such as Sample Return missions, entry probes to Venus, and even Mars. Given that TPS is a cross-cutting technology requiring specialized resources in terms of expertise, facilities, and capabilities across NASA and industry, the specific recommendations made above are applicable for all solar system destinations to an atmospheric body. These investments will thus provide maximum return to NASA's future missions.

Finally, the TPS community requests early participation in future atmospheric entry mission studies commissioned by the Decadal panels, in order to advise about material feasibility and performance, and potential mission constraints.

\section{REFERENCES}

[1] Venkatapathy, E., et al., "Thermal Protection System Technologies for Enabling Future Outer Planet Missions", White paper to the NRC decadal survey outer planets sub-panel, 2008.

[2] Venkatapathy, E., et al., "Thermal Protection System Technologies for Enabling Future Venus Exploration”, White paper to the NRC decadal survey inner planets sub-panel, 2008.

[3] Venkatapathy, E., et al., "Entry System Technology Readiness for Ice-Giant Probe Missions", Space Sci Rev (2020) 216:22

[4] "Ice Giants Pre-Decadal Study Final Report", June 2017, JPL D-100520

[5] Dutta, S., et al., "Aerocapture as an Option for Ice Giants Missions", White paper for the planetary decadal survey 2023-2032.

[6] Venkatapathy, E., et al.," Thermal Protection System to Enable Ice Giant Aerocapture Mission for Delivering both an Orbiter and an In-Situ Probe", White paper for the planetary decadal survey 2023-2032.

[7] Gasch, M., et al., "Technologies for Future Venus Exploration”, White paper for the planetary decadal survey 2023-2032.

[8] Venkatapathy, E., et al., "Sustaining Mature Thermal Protection Systems Crucial for Future In-Situ Planetary Missions", White paper for the planetary decadal survey 2023-2032.

[9] MacDonald, M., et al., "NASA Ames Thermophysics Ground Test Facilities Supporting Future Planetary Atmospheric Entry", White paper for the planetary decadal survey 2023-2032.

[10] Wilder, M. C., et al., "Effects of Distributed Surface Roughness on Turbulent Heat Transfer Augmentation Measured in Hypersonic Free Flight," AIAA-2014-0512, 52nd AIAA Aerospace Sciences Meeting, 13-17 January 2014.

[11] Wilder, M. C. and Prabhu, D. K., "Rough-Wall Turbulent Heat Transfer Experiments in Hypersonic Free Flight," AIAA-2019-3009, AIAA Aviation 2019 Forum, 17-21 June 2019, Dallas, Texas.

[12] Santos, J., et al., "Entry, Descent, and Landing Instrumentation", White paper for the planetary decadal survey 2023-2032. 